\title{
Essentiality of FASII pathway for Staphylococcus aureus
}

\author{
Arising from: S. Brinster et al. Nature 458, 83-86 (2009)
}

Recently, Brinster et al. ${ }^{1}$ suggested that type II fatty-acid biosynthesis (FASII) is not a suitable antibacterial target for Gram-positive pathogens because they use fatty acids directly from host serum rather than de novo synthesis. Their findings, if confirmed, are relevant for further scientific and financial investments in the development of new drugs targeting FASII. We present here in vitro and in vivo data demonstrating that their observations do not hold for Staphylococcus aureus, a major Gram-positive pathogen causing several human infections. The observed differences among Gram-positive pathogens in FASII reflects heterogeneity either in fatty-acid synthesis or in the capacity for fatty-acid uptake from the environment.

The effect of serum in downregulating FASII in S.agalactiae observed by Brinster et al. ${ }^{1}$ suggests redundancy in fatty-acid synthesis upon exposure to a fatty-acid-rich environment. We confirmed that serum leads to a downregulation of S. agalactiae fab genes, but demonstrated unaltered $f a b$ expression in $S$. aureus (Fig. 1a). This might be due to differences in exogenous fatty-acid uptake, for example, it may be driven by cell-membrane hydrophobicity. S. aureus adapts its membrane hydrophobicity to escape innate-immune molecules, such as antimicrobial peptides and amphipathic fatty acids ${ }^{2,3}$, reiterating the specificity of host-pathogen interactions ${ }^{4,5}$.

Validating the effect of exogenous fatty acids for S. aureus requires inactivation of the drug target. Knockdown of enoyl-acyl-carrierprotein (ACP) reductase (FabI), a key enzyme in S. aureus FASII, was achieved by expression of tetracycline-induced fabI antisense RNA, which has previously been shown to have target selectivity ${ }^{6}$. In contrast to S. agalactiae ${ }^{1}$, we observed that fabI downregulation severely compromised S.aureus growth in both the absence and presence of serum (Fig. 1b and c), Tween-80 and oleic and stearic fatty acids (data not shown). To show whether $S$. aureus can adapt to serum fatty acid, we pre-incubated the fabI antisense strain for 12 hours in $50 \%$ serum. Even after this long fatty-acid exposure, the fabI antisense strain was growth-compromised in 50\% serum (Fig. 1b). These differences can be explained by mechanistic and physiological diversity among bacterial FASII enzymes ${ }^{7}$. Whereas streptococci have FabK as the ACP reductase, staphylococci possess FabI as the fatty-acid elongation counterpart ${ }^{7}$. Additionally, only streptococci possess FabM, a cis-trans isomerase, suggesting that they have a different mechanism for formation of fatty acids ${ }^{8,9}$.

Brinster et al. ${ }^{1}$ reported highly increased minimum inhibitory concentrations (MICs) in the presence of serum for hydrophobic compounds like cerulenin (which targets $\mathrm{FabB} / \mathrm{FabH}$ ) and triclosan (targeting FabI) for several Gram-positive pathogens ${ }^{10,11}$. We tested the inhibitory activities of these compounds, and also platensimycin ${ }^{12}$ (targeting FabF/FabB) and CG400549 (targeting FabI; ref. 13). All inhibitors lost activity completely against S.agalactiae when these bacteria were grown in the presence of serum or Tween-80 (Table 1), suggesting exogenous serum compensatory mechanisms. However, for S. aureus serum or Tween-80 had little impact on the MICs of FASII inhibitors. The slightly increased MIC values for triclosan are due to its significant plasma protein binding (about 99.5\%) (Table 1). Furthermore, pre-incubation with exogenous fatty acids did not rescue the growth inhibition of S. aureus by CG400549 (data not shown). To determine whether serum inhibits cellular fatty-acid synthesis, we measured the incorporation of $\left[1-{ }^{14} \mathrm{C}\right]$-acetate into membrane fatty acids. Serum had no significant effect on $\left[1-{ }^{14} \mathrm{C}\right]$-acetate incorporation in $S$. aureus, suggesting that these bacteria continue fatty-acid synthesis even in the presence of serum. In contrast, for S. agalactiae we observed
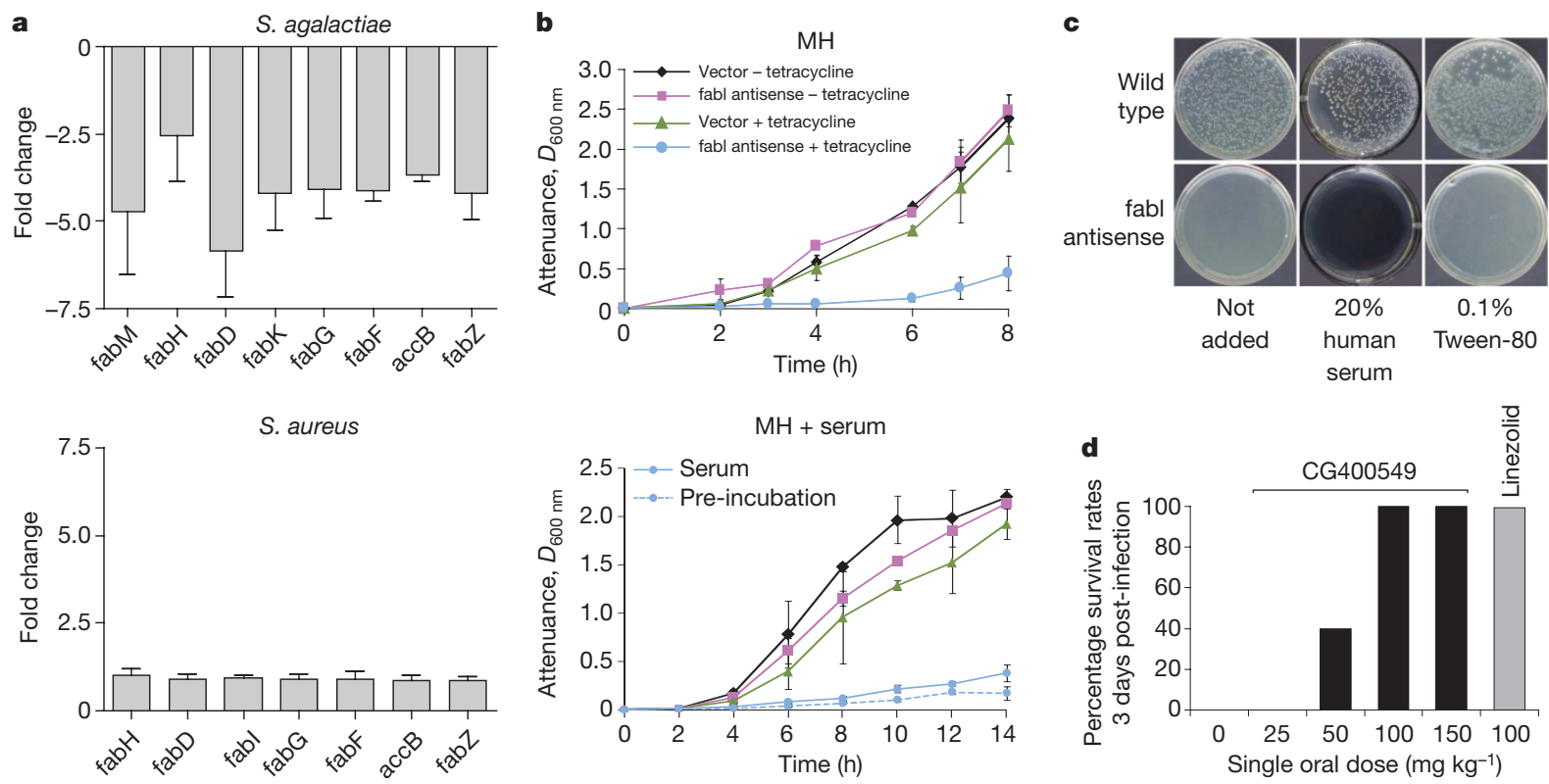

Figure 1 | S. aureus FASII inhibition is not rescued by exogenous fatty acids. a, Transcription levels of $f a b$ genes in mid-logarithmic S. agalactiae (left panel) in Todd Hewitt medium and S. aureus (right panel) in Mueller Hinton (MH) medium, with or without $50 \%$ human serum. The fold change was calculated using the standard curve method with $g y r A$ as control (average \pm s.d.; $n=4$ ). b, Growth of wild-type (empty pAJ96 vector) and fabI antisense $S$. aureus strains in liquid MH with and without $150 \mathrm{ng} \mathrm{ml}^{-1}$ tetracycline (left panel) and $\mathrm{MH}+50 \%$ human serum with and without $250 \mathrm{ng} \mathrm{ml}^{-1}$ tetracycline (right panel) $(n=2)$. The right panel also shows

growth of $f a b I$ antisense $S$. aureus (induced with $250 \mathrm{ng} \mathrm{ml}^{-1}$ tetracycline) with 12-hour serum pre-adaptation (dotted blue line) $(n=2)$. Values are represented as averages \pm s.d. c, Growth of wild-type (empty pAJ96 vector) and $f a b I$ antisense strains on solid medium with $20 \%$ human serum or $0.1 \%$ Tween- 80 and incubated for 24 hours at $37^{\circ} \mathrm{C}$. FabI antisense is induced by adding $300 \mathrm{ng} \mathrm{ml}^{-1}$ tetracycline (MH + Tween-80) and $500 \mathrm{ng} \mathrm{ml}^{-1}$ tetracycline ( $\mathrm{MH}+20 \%$ human serum). d, In vivo activity of CG400549 against $S$. aureus in mouse peritonitis model. Percentage survival is shown after single oral dose (positive control is linezolid). 
Table 1 | Susceptibilities of S. aureus and S. agalactiae to FASII pathway inhibitors

\begin{tabular}{|c|c|c|c|c|c|c|c|}
\hline & \multicolumn{3}{|c|}{ MIC of S. aureus ATCC $29213\left(\mu \mathrm{g} \mathrm{ml}^{-1}\right)$} & \multicolumn{3}{|c|}{ MIC of S. agalactiae NEM316 $\left(\mu \mathrm{g} \mathrm{ml}^{-1}\right)$} & \multirow[t]{2}{*}{ Plasma protein binding (mean \pm s.d.) } \\
\hline & No addition & $10 \%$ human serum & $0.1 \%$ Tween-80 & No addition & $10 \%$ human serum & $0.1 \%$ Tween-80 & \\
\hline Triclosan & 0.125 & 1 & 2 & 8 & $>64$ & $>64$ & $>99.5 \%$ \\
\hline Cerulenin & 128 & 256 & 256 & 32 & $>512$ & $>512$ & Not determined \\
\hline Platensimycin & 2 & 2 & 2 & 8 & $>64$ & $>64$ & $51.25 \% \pm 2.76 \%$ \\
\hline CG400549 & 0.25 & 0.5 & 1 & $>64$ & $>64$ & $>64$ & $94.35 \% \pm 0.46 \%$ \\
\hline
\end{tabular}

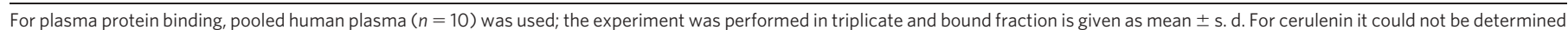

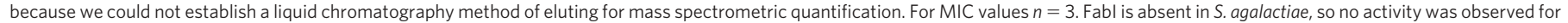
CG400549.

a significant decrease in $\left[1-{ }^{14} \mathrm{C}\right]$ - acetate incorporation in the presence of serum (data not shown). These results suggest functional differences in FASII pathways among different Gram-positive pathogens.

Final validation of drug targets is accomplished by in vivo pharmacodynamics. We tested CG400549 in a mouse peritonitis model and observed a dose-dependent survival of mice (Fig. 1d). Brinster et al. ${ }^{1}$ pointed out that bacterial adaptation to serum fatty acid is a gradual process. We observed that dosing of CG400549 in neutropenic mice, 6 hours after systemic infection (which allows host adaptation), killed about $\log _{10}$ [colony-forming units $]=1.7 \pm 0.3$ in infected spleens (in untreated controls no bacteria were killed). These results and earlier reports on the efficacy of FabI inhibitors in rodent models ${ }^{14}$, including the clinical efficacy of Isoniazid, a FASII inhibitor in Mycobacterium tuberculosis ${ }^{15}$, argue against the broad claims by Brinster et al. ${ }^{1}$ that FASII is not a pharmaceutically attractive target.

\section{METHODS}

S. aureus ATCC29213 and S. agalactiae NEM316 strains were used. Expression levels of FASII genes and the control gyrA were determined using quantitative real-time polymerase chain reaction (PCR). FabI antisense ${ }^{6}$ was cloned into the tetracycline-inducible pAJ96 vector (A. Neill, University of Leeds, UK) and electroporated into RN4220(pSTE2) (T. Hauschild, University of Bialystok, Poland). Swiss mice were intraperitoneally infected with S. aureus $\left(10^{8}\right.$ colonyforming units) and immediately treated orally with CG400549 (percentage survival). Neutropenic mice were infected with S. aureus and dosed orally 6 hours post-infection with CG400549 (three times a day at $100 \mathrm{mg} \mathrm{kg}^{-1}$ ) for two days and killed to determine spleen colony-forming unit counts. References were purchased from Fasgen (triclosan), Sequoia Products (linezolid/cerulenin), Interchim (CG400549) and BioAustralis (platensimycin).

Wendy Balemans ${ }^{1}$, Nacer Lounis ${ }^{1}$, Ron Gilissen ${ }^{2}$, Jerome Guillemont ${ }^{3}$, Kenny Simmen ${ }^{4}$, Koen Andries ${ }^{1} \&$ Anil Koul $^{1}$

${ }^{1}$ Department of Antimicrobial Research, Tibotec BVBA, Johnson \& Johnson, Turnhoutseweg 30, B-2340 Beerse, Belgium.

e-mail: akoul@its.jnj.com

2Department of ADMETox, Pharmaceutical Research and Development, Johnson \& Johnson, Turnhoutseweg 30, B-2340 Beerse, Belgium. ${ }^{3}$ Department of Antimicrobial Research, Centre de Recherche Janssen-Cilag, Johnson \& Johnson, Campus de Maigremont BP615, F-27106 Val de Reuil cedex, France.
${ }^{4}$ Department of Antimicrobial Research, Tibotec BVBA, Johnson \& Johnson, Gen. De Wittelaan L 11B 3, B-2800 Mechelen, Belgium.

Received 18 September; accepted 12 October 2009.

1. Brinster, S. et al. Type II fatty acid synthesis is not a suitable antibiotic target for Grampositive pathogens. Nature 458, 83-86 (2009).

2. Weidenmaier, C. et al. DltABCD- and MprF-mediated cell envelope modifications of Staphylococcus aureus confer resistance to platelet microbicidal proteins and contribute to virulence in a rabbit endocarditis model. Infect. Immun. 73, 8033-8038 (2005).

3. Peschel, A. et al. Staphylococcus aureus resistance to human defensins and evasion of neutrophil killing via the novel virulence factor MprF is based on modification of membrane lipids with I-lysine. J. Exp. Med. 193, 1067-1076 (2001).

4. Koul, A., Herget, T., Klebl, B. \& Ullrich, A. Interplay between mycobacteria and host signalling pathways. Nature Rev. Microbiol. 2, 189-202 (2004).

5. Finlay, B. B. \& Cossart, P. Exploitation of mammalian host cell functions by bacterial pathogens. Science 276, 718-725 (1997).

6. Ji, Y. et al. Validation of antibacterial mechanism of action using regulated antisense RNA expression in Staphylococcus aureus. FEMS Microbiol. Lett. 231, 177-184 (2004).

7. Marrakchi, H., Zhang, Y. M. \& Rock, C. O. Mechanistic diversity and regulation of Type II fatty acid synthesis. Biochem. Soc. Trans. 30, 1050-1055 (2002).

8. Altabe, S., Lopez, P. \& de Mendoza, D. Isolation and characterization of unsaturated fatty acid auxotrophs of Streptococcus pneumoniae and Streptococcus mutans. J. Bacteriol. 189, 8139-8144 (2007).

9. Zhang, Y. M. \& Rock, C. O. Membrane lipid homeostasis in bacteria. Nature Rev. Microbiol. 6, 222-233 (2008).

10. Omura, S. Cerulenin. Methods Enzymol. 72, 520-532 (1981)

11. Heath, R. J., White, S. W. \& Rock, C. O. Lipid biosynthesis as a target for antibacterial agents. Prog. Lipid Res. 40, 467-497 (2001).

12. Wang, J. et al. Platensimycin is a selective FabF inhibitor with potent antibiotic properties. Nature 441, 358-361 (2006).

13. Yum, J. H. et al. In vitro activities of CG400549, a novel Fabl inhibitor, against recently isolated clinical staphylococcal strains in Korea. Antimicrob. Agents Chemother. 51, 2591-2593 (2007).

14. Payne, D. J. et al. Discovery of a novel and potent class of Fabl-directed antibacterial agents. Antimicrob. Agents Chemother. 46, 3118-3124 (2002).

15. Vilcheze, C. et al. Transfer of a point mutation in Mycobacterium tuberculosis inhA resolves the target of isoniazid. Nature Med. 12, 1027-1029 (2006).

Author Contributions W.B., N.L., R.G. and J.G. contributed to the experimental work. A.K., W.B., N.L., R.G., K.A. and K.S. contributed to the project planning, study design and data analysis. W.B. and A.K. wrote the manuscript.

Competing financial interests: all authors are members of an antimicrobial drug discovery group working for a pharmaceutical company.

doi:10.1038/nature08667

\section{Brinster et al. reply}

\section{Replying to: W. Balemans et al. Nature 462, doi:10.1038/nature08668 (2009).}

Our studies led us to conclude that growth of major Gram-positive pathogens, including Staphylococcus aureus, is not inhibited by FASIItargeted antibiotics in septicaemic infection, owing to compensation by serum fatty acids ${ }^{1}$. The comments of Balemans et al. ${ }^{2}$ challenge the generality of our results, mainly on the basis of their own work, which is aimed at developing FabI inhibitors for treatment of S. aureus infections. Their allusion to the documented use of FASII inhibitors to treat mycobacterial infections is misleading. Mycobacteria were not considered in our study, because (1) their main route of pathogenesis is not sepsis, and (2) they require mycolic acids for normal growth, which are lacking in serum. The results we present here further reinforce the conclusions of our article.

To assess directly the conclusions of Balemans et al. about the use of S. aureus FabI as an antimicrobial target, we performed experiments using the same system. S. aureus strain RN4220 was pre-grown in Todd Hewitt medium $(\mathrm{TH})$ or in $\mathrm{TH}+0.015 \%$ linoleic acid (C18:2; prepared from $0.15 \%$ stock in $5 \%$ bovine serum albumin). Overnight cultures were diluted in the respective media, and divided 


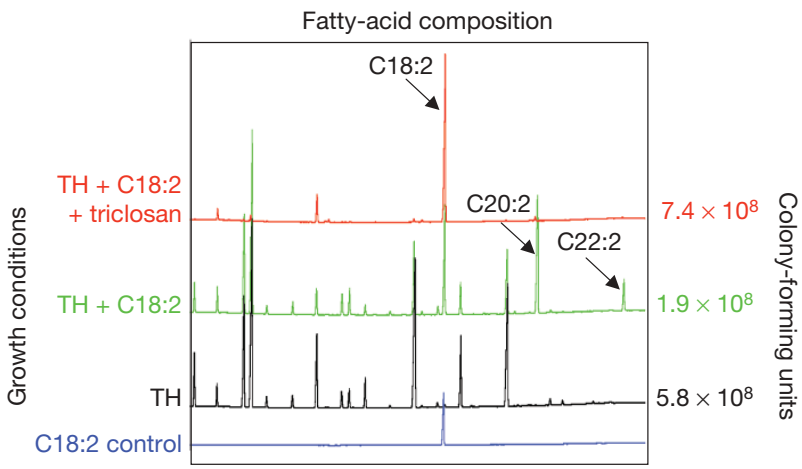

Figure 1 S. aureus uses exogenous fatty acids, and grows despite FASI inhibition by triclosan. S. aureus RN4220 was grown overnight in TH medium, or in $\mathrm{TH}$ medium containing $0.015 \% \mathrm{C} 18: 2$. Cultures were diluted $1: 100$ in the same media. Each culture was then divided into two equal parts, and triclosan $\left(4 \mu \mathrm{g} \mathrm{ml}^{-1}\right.$ final concentration) was added to one aliquot of the $\mathrm{TH}$ culture, and one aliquot of the TH $+\mathrm{C} 18: 2$ culture. After 12 hours, whole-cell fatty acids were analysed by gas chromatography. Profiles show the pure C18:2 control (blue line), and S. aureus grown in TH (black line), in $\mathrm{TH}+\mathrm{C} 18: 2$ (green line) and in $\mathrm{TH}+\mathrm{C} 18: 2+$ triclosan (red line). We note that S. aureus grown in C18:2 (green line) shows de novo synthesis of two novel peaks (arrows), corresponding to $\mathrm{C} 20: 2$ and C22:2. Growth is given as the colony-forming units of the three analysed cultures.

into two aliquots, either without antibiotic, or with $4 \mu \mathrm{g} \mathrm{ml}^{-1}$ triclosan (FabI-specific inhibitor ${ }^{3}$ ), and further incubated for 12 hours. As expected, no growth was obtained in the TH culture containing triclosan. Fatty-acid profiles of whole-cell extracts of the remaining cultures ( $\mathrm{TH}, \mathrm{TH}+\mathrm{C} 18: 2$ and $\mathrm{TH}+\mathrm{C} 18: 2+$ triclosan) were determined by gas chromatography and bacteria were counted (Fig. 1). Pure C18:2 used as control produced a single gas chromatography peak (blue line). S. aureus grown in $\mathrm{TH}$ (black line) generated a complex profile that lacks C18:2. In contrast, C18:2 is prominent in the complex fatty-acid profile of cells grown in TH plus C18:2 (green line). Significantly, C18:2 appears to be used as a substrate on which to synthesize new fatty acids: C20:2 and C22:2 (arrows). When C18:1 is added to cultures instead of C18:2 (results not shown), the synthesized products were C20:1 and C22:1. This shows that $S$. aureus incorporates exogenous fatty acids, as suggested from previous studies ${ }^{4,5}$. Moreover, the fatty-acid profile displays the exogenously added fatty acids, as well as endogenous fatty acids, consistent with continued fab gene expression in S. aureus, as determined by Balemans et al. ${ }^{2}$ (their figure 1a).

When triclosan is added to the S. aureus culture grown in TH plus C18:2 (red line), fatty-acid synthesis is blocked: the C18:2 peak is still prominent, but peaks corresponding to fatty acids synthesized by S. aureus are strongly diminished. The C18:2-derived peaks, C20:2 and C22:2, are not detected. We concluded that triclosan reaches its target in the presence of fatty acids, and blocks bacterial fatty-acid synthesis. Bacterial counts (colony-forming units) show that FASII inhibition by triclosan does not stop $S$. aureus growth when exogenous C18:2 is provided. Similar results were obtained when cerulenin was used rather than triclosan, or when oleic acid or 100\% serum was used as exogenous fatty acids (not presented).

These new results prove that FASII-directed antimicrobials can indeed reach their targets when exogenous fatty acids are present, to inhibit fatty-acid synthesis in S. aureus. However, FASII inhibition is compensated by the exogenous fatty acids, and allows bacterial growth. These results strengthen our initial hypothesis, and its relevance for S. aureus. Importantly, we succeeded in obtaining a confirmed S. aureus fabI deletion mutant by maintaining bacteria on a fatty-acid source throughout construction steps (S.B., G.L., C.P., A.G. and P.T.-C., manuscript in preparation). These findings further substantiate the generality of our study, and support our conclusions that the antibacterial activity of FASII inhibitors would be compromised in the treatment of S. aureus septicaemic infections.

Sophie Brinster ${ }^{1,2}$, Gilles Lamberet ${ }^{3}$, Bart Staels ${ }^{4}$, Patrick Trieu-Cuot ${ }^{5}$, Alexandra Gruss ${ }^{3}$ \& Claire Poyart ${ }^{1,2,5,6}$

${ }^{1}$ Institut Cochin, Université Paris Descartes, CNRS (UMR 8104), 75014

Paris, France.

e-mail: claire.poyart@cch.aphp.fr

${ }^{2}$ INSERM, U567, 75014, Paris, France.

${ }^{3}$ INRA U888, Unité des Bactéries Lactiques et Pathogènes

Opportunistes, 78352 Jouy en Josas, France.

e-mail: alexandra.gruss@jouy.inra.fr

${ }^{4}$ Institut Pasteur de Lille, INSERM UMR545, Université Lille 2, 59019 Lille, France.

${ }^{5}$ Institut Pasteur, Unité de Biologie des Bactéries Pathogènes à Gram Positif, URA CNRS 2172, 75015 Paris, France.

${ }^{6}$ Assistance Publique Hôpitaux de Paris, Centre National de Référence des Streptocoques, Hôpital Cochin, 75014, Paris, France.

1. Brinster, S. et al. Type Il fatty acid synthesis is not a suitable antibiotic target for Grampositive pathogens. Nature 458, 83-86 (2009).

2. Balemans, W. et al. Essentiality of FASIl pathway for Staphylococcus aureus. Nature 462, doi:10.1038/nature08668 (this issue).

3. Heath, R. J. \& Rock, C. O. Fatty acid biosynthesis as a target for novel antibacterials. Curr. Opin. Investig. Drugs 5, 146-153 (2004).

4. Altenbern, R. A. Cerulenin-inhibited cells of Staphylococcus aureus resume growth when supplemented with either a saturated or an unsaturated fatty acid. Antimicrob. Agents Chemother. 11, 574-576 (1977).

5. Weerkamp, A. \& Vogels, G. D. Physiological conditions affecting Staphylococcus aureus susceptibility to staphylococcin 1580. Antimicrob. Agents Chemother. 13, 146-153 (1978).

doi:10.1038/nature08668 